\title{
Institutional review of the management of type II odontoid fractures: associations and outcomes with fibrous union
}

\author{
Christopher Wilson, MD, ${ }^{1}$ Mariana Hoyos, MD, ${ }^{1}$ Andrew Huh, MD, ${ }^{1}$ Blake Priddy, MD, ${ }^{1}$ \\ Stephen Avila, MBS, ${ }^{1}$ Stephen Mendenhall, MD, ${ }^{1}$ Miracle C. Anokwute, MD, ${ }^{1}$ \\ George J. Eckert, MAS, ${ }^{2}$ and David W. Stockwell, MD' \\ Departments of ${ }^{1}$ Neurological Surgery and ${ }^{2}$ Biostatistics, Indiana University School of Medicine, Indianapolis, Indiana
}

\begin{abstract}
OBJECTIVE Type II odontoid fractures may be managed operatively or nonoperatively. If managed with bracing, bony union may never occur despite stability. This phenomenon is termed fibrous union. The authors aimed to determine associations with stable fibrous union and compare the morbidity of patients managed operatively and nonoperatively.

METHODS The authors performed a retrospective review of their spine trauma database for adults with type ll odontoid fractures between 2015 and 2019. Two-sample t-tests and Fisher's exact tests identified associations with follow-up stability and were used to compare operative and nonoperative outcomes. Sensitivity, specificity, and predictive values were calculated to validate initial stable upright cervical radiographs related to follow-up stability.
\end{abstract}

RESULTS Among 88 patients, $10 \%$ received upfront surgical fixation, and $90 \%$ were managed nonoperatively, of whom $22 \%$ had fracture instability on follow-up. Associations with instability after nonoperative management include myelopathy (OR $0.04,95 \% \mathrm{Cl} 0.0-0.92$ ), cerebrovascular disease (OR $0.23,95 \% \mathrm{Cl} 0.06-1.0$ ), and dens displacement $\geq 2$ $\mathrm{mm}(\mathrm{OR} 0.29,95 \% \mathrm{Cl} 0.07-1.0)$. Advanced age was not associated with follow-up instability. Initial stability on upright radiographs was associated with stability on follow-up (OR 4.29, 95\% $\mathrm{Cl} 1.0-18$ ) with excellent sensitivity and positive predictive value (sensitivity $89 \%$, specificity $35 \%$, positive predictive value $83 \%$, and negative predictive value $46 \%$ ). The overall complication rate and respiratory failure requiring ventilation on individual complication analysis were more common in operatively managed patients ( $33 \% \mathrm{vs} 3 \%$, respectively; $p=0.007$ ), even though they were generally younger and healthier than those managed nonoperatively. Operative or nonoperative management conferred no difference in length of hospital or ICU stay, discharge disposition, or mortality.

CONCLUSIONS The authors delineate the validity of upright cervical radiographs on presentation in association with follow-up stability in type II odontoid fractures. In their experience, factors associated with instability included cervical myelopathy, cerebrovascular disease, and fracture displacement but not increased age. Operatively managed patients had higher complication rates than those managed without surgery. Fibrous union, which can occur with nonoperative management, provided adequate stability.

https://thejns.org/doi/abs/10.3171/2020.8.SPINE20860

KEYWORDS type II odontoid fracture; fibrous union; stability; external orthosis; cervical; trauma

$\mathrm{T}$ HE odontoid process is a superior extension from the body of the axis that articulates with the atlas. Immediately superior to the axis vertebral body, the odontoid diameter is its narrowest. Fracture at this vulnerable portion of the odontoid process was termed a type II odontoid fracture by Anderson and D'Alonzo. ${ }^{1}$ The most common axis fracture is the type II odontoid fracture., ${ }^{1,2}$ It is vulnerable to nonunion resulting from vascular watershed of the fracture fragment, prominent bone density loss in the elderly at the fracture site, minimal cancellous bone surface area along the fracture line, and torque imposed by the atlantoaxial ligaments. ${ }^{2-12}$ Contention exists regarding the management of type II odontoid fractures. Previous concern regarding the potential for devastating neurological injury if the fracture is not adequately stabilized has prompted some to advocate early surgical stabilization. ${ }^{1,2,5,8,13}$ One consequence of an uncomplicated surgical fixation of type II odontoid fractures is restricted

ABBREVIATIONS LOS = length of stay; NPV = negative predictive value; PPV = positive predictive value; $Q O L$ = quality of life.

SUBMITTED May 12, 2020. ACCEPTED August 11, 2020.

INCLUDE WHEN CITING Published online January 22, 2021; DOI: 10.3171/2020.8.SPINE20860. 
neck mobility and development of neck pain, which can reduce a patient's quality of life (QOL) ${ }^{3,14}$ Additionally, operative risks have been cited as a reason to use nonoperative therapy, particularly in elderly patients who are more prone to complications after surgery., ${ }^{1,6,13-15}$ The use of contemporary rigid cervical orthoses has improved adverse event profiles relative to traditional orthoses, including halo vests. ${ }^{1,8,16,17}$ There is a paucity of evidence favoring either surgical or nonsurgical management of type II odontoid fractures. Regardless of the management strategy employed, the goal of treatment is expeditious fracture stabilization and union. When this does not occur, it is termed a "nonunion." Increasing age, fracture displacement or angulation, myelopathy, and transverse ligament disruption have been previously associated with fracture nonunion. ${ }^{2,4}$ However, many studies have reported these risk factors with respect to bony union, which occurs when bony trabeculae bridge the gap along the fracture. Bony union does not always happen, and $75 \%$ of fractures without bony union may be stable. ${ }^{8,12,14}$ This phenomenon is termed "fibrous union." Fibrous union may be accomplished safely without surgery in many cases, which eliminates previously reported surgical risks. ${ }^{6}$ Consequently, at our institution, we often manage patients with type II odontoid fractures nonoperatively when fracture stability is demonstrated on upright radiographs with the patient in a brace. We aimed to evaluate factors associated with stable fibrous union, assess the value of initial upright cervical radiographs in association with follow-up stability, and compare outcomes of operative and nonoperative management of type II odontoid fractures.

\section{Methods}

We conducted an institutional review board-approved retrospective review of our spine trauma database, which includes two level 1 trauma centers. We queried the database for adults (age $\geq 18$ years) who sustained acute type II odontoid fractures between the years of 2015 and 2019 and underwent operative or nonoperative management. Patients included in the search must have had a cervical spine CT scan at the time of presentation, followed by at least two upright cervical radiographs in a cervical collar. One upright cervical radiograph was obtained at initial presentation, and additional radiographs were obtained during outpatient follow-up. All cervical radiographs were completed with the patients upright and included anteroposterior, lateral, and open-mouth odontoid views. Patients without follow-up imaging beyond their initial presentation or who died prior to follow-up were excluded. Initial upright cervical radiographs were used to assess fracture stability on presentation. Stability on initial upright anteroposterior, lateral, and open-mouth odontoid cervical radiographs was defined as no spondylolisthesis more than $2 \mathrm{~mm}$ in any direction with the patient wearing a cervical collar using the methods of Bono et al. on lateral imaging and measuring the overhang of the fracture fragment on the proximal odontoid on anteroposterior and open-mouth odontoid views. ${ }^{18}$ Later, upright radiographs were used to assess follow-up stability at monthly intervals for 3 months using the same definition of stability, at which point flexion-extension radiographs were evaluated. Flexion-extension radiographs were deemed to demonstrate stability so long as no motion between the fracture fragment and adjacent bone of more than $2 \mathrm{~mm}$ was present when comparing flexion and extension radiographs using the same reference lines as Bono et al..$^{18}$ Examples of initial and follow-up radiographs showing stability are shown in Fig. 1. If the fracture was stable at 3 months, the cervical collar was removed, and the patients were managed expectantly thereafter with as-needed follow-up. The decision regarding initial operative or nonoperative management was at the discretion of the treating attending neurosurgeon. However, patients with fracture stability on their initial upright radiographs as determined by the associated radiologist were managed nonoperatively. Nonoperative intervention included a rigid cervical orthosis worn continually. No halo fixation was used. A clinical flow diagram in Fig. 2 illustrates the management at our institution. Bony union was diagnosed if bony trabeculae were visible spanning the fracture. Otherwise, patients were considered to have fibrous union. The length of follow-up was calculated as the time between the day of presentation and the date of the last clinic contact or cervical spine imaging. All surgical patients underwent posterior cervical or occipitocervical instrumented fusion using image guidance followed by stabilization with a rigid cervical orthosis for at least 4 weeks.

We recorded a multitude of factors possibly associated with fracture nonunion. These included all components of the Charlson Comorbidity Index, prior cervical spine surgery, mechanism of injury, and tobacco use. ${ }^{19}$ Physical examination findings including neurological deficit or myelopathy were evaluated. Finally, we examined the relationship between radiographic parameters, including odontoid displacement, angulation, and comminution, and stability on follow-up imaging. Fisher's exact test was used to determine the significance of the relationship between each factor and cervical spine instability. A 95\% confidence interval for odds ratios was computed, and $\mathrm{p}<$ 0.05 was considered statistically significant.

The value of stability of initial upright cervical radiographs in relation to follow-up stability was assessed using Fisher's exact test. We calculated sensitivity, specificity, positive predictive value (PPV), and negative predictive value (NPV) for the use of the initial stable upright cervical radiographs in relation to follow-up stability.

Outcomes of nonoperatively and operatively managed patients were compared. These included ICU length of stay (LOS), hospital LOS, high risk medical complications (e.g., myocardial infarction, deep venous thrombosis, pulmonary embolism, urinary tract infection, acute kidney injury, respiratory failure), discharge disposition, and mortality within 30 days of presentation. Delayed neurological and nonneurological adverse events were defined as neurological deterioration and nonneurological complications occurring more than 30 days after injury if managed nonoperatively and more than 30 days postoperatively if managed operatively. Two-sample t-tests and Fisher's exact tests were used to compare differences in these outcomes between patients managed with upfront surgery and those with initial bracing. A 95\% confidence 


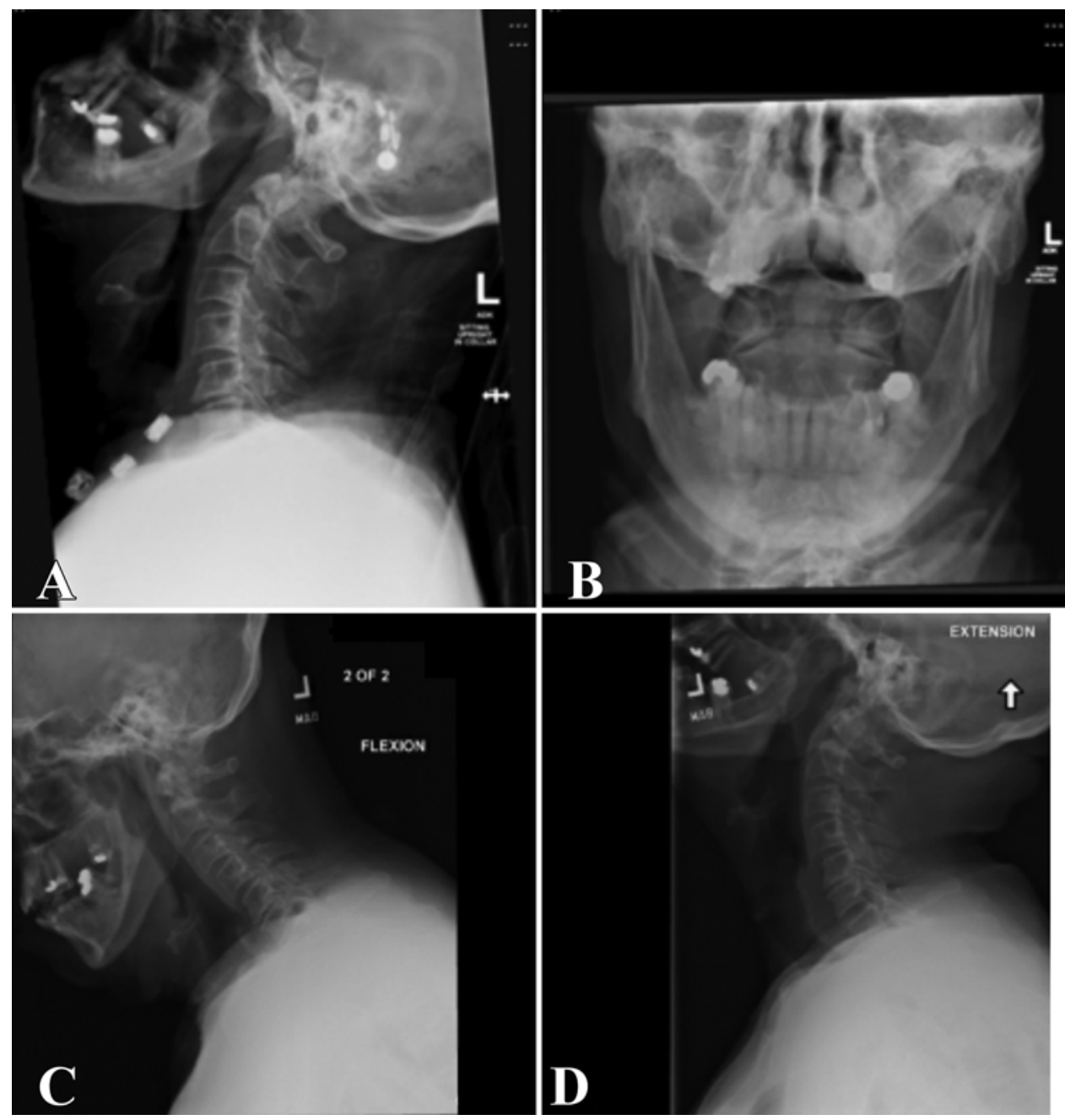

FIG. 1. A and B: Lateral (A) and anteroposterior open-mouth odontoid (B) initial upright radiographs showing initial stability. C and D: Flexion (C) and extension (D) radiographs obtained 3 months after injury, showing that stability has been maintained.

interval for the odds ratio was also computed for binary outcomes. A 5\% significance level was used. All statistical analyses were performed using SAS version 9.4 (SAS Institute Inc.).

\section{Results}

Ninety-nine patients sustained type II odontoid fractures and were evaluated by our neurosurgery service with a mean follow-up of 5.2 months (range 1 day-48 months). Table 1 summarizes baseline demographics. The mean patient age was 80 years and was higher in the nonoperative group than in the group managed with early surgical fixation (82 years vs 65 years, $\mathrm{p}<0.05)$. Eleven of the 99 patients had insufficient data for follow-up and were excluded. Nine $(10 \%)$ of the remaining 88 patients required early surgical intervention and $79(90 \%)$ were treated nonoperatively with a rigid cervical brace for 3 months. Myelopathy was more common among those managed with initial surgical fixation $(22 \%$ vs $2.5 \%$, p = $0.05)$. Similarly, the mean length of fracture displacement was greater in patients receiving upfront surgery (mean $6.75 \mathrm{~mm}$ vs $1.75 \mathrm{~mm}, \mathrm{p}=0.001$ ). The direction of displacement and comminution were similar between groups, as was the angulation (mean $12.3^{\circ}$ vs $8.1^{\circ}, \mathrm{p}=$ 0.57 ). There was no significant difference in trauma severity, tobacco use, diabetes, cerebrovascular disease, or renal disease between patients managed operatively and those managed nonoperatively.

Initial upright cervical radiographs in a hard cervical brace were obtained in all 79 patients who were nonoperatively managed during their presenting encounter. Among the nonoperative cohort, $66(83.5 \%)$ of the fractures were stable on initial upright radiographs. Among those with stability on the initial upright radiographs, $11(16.7 \%)$ had instability on follow-up imaging. Two of the 11 patients in whom delayed instability developed underwent subsequent surgical fixation, whereas 5 were managed with a cervical collar indefinitely, and 4 patients entered hospice care without continued cervical immobilization. Two of those managed with a chronic cervical collar were offered surgery but refused.

Of the 13 patients with instability on initial evalua- 


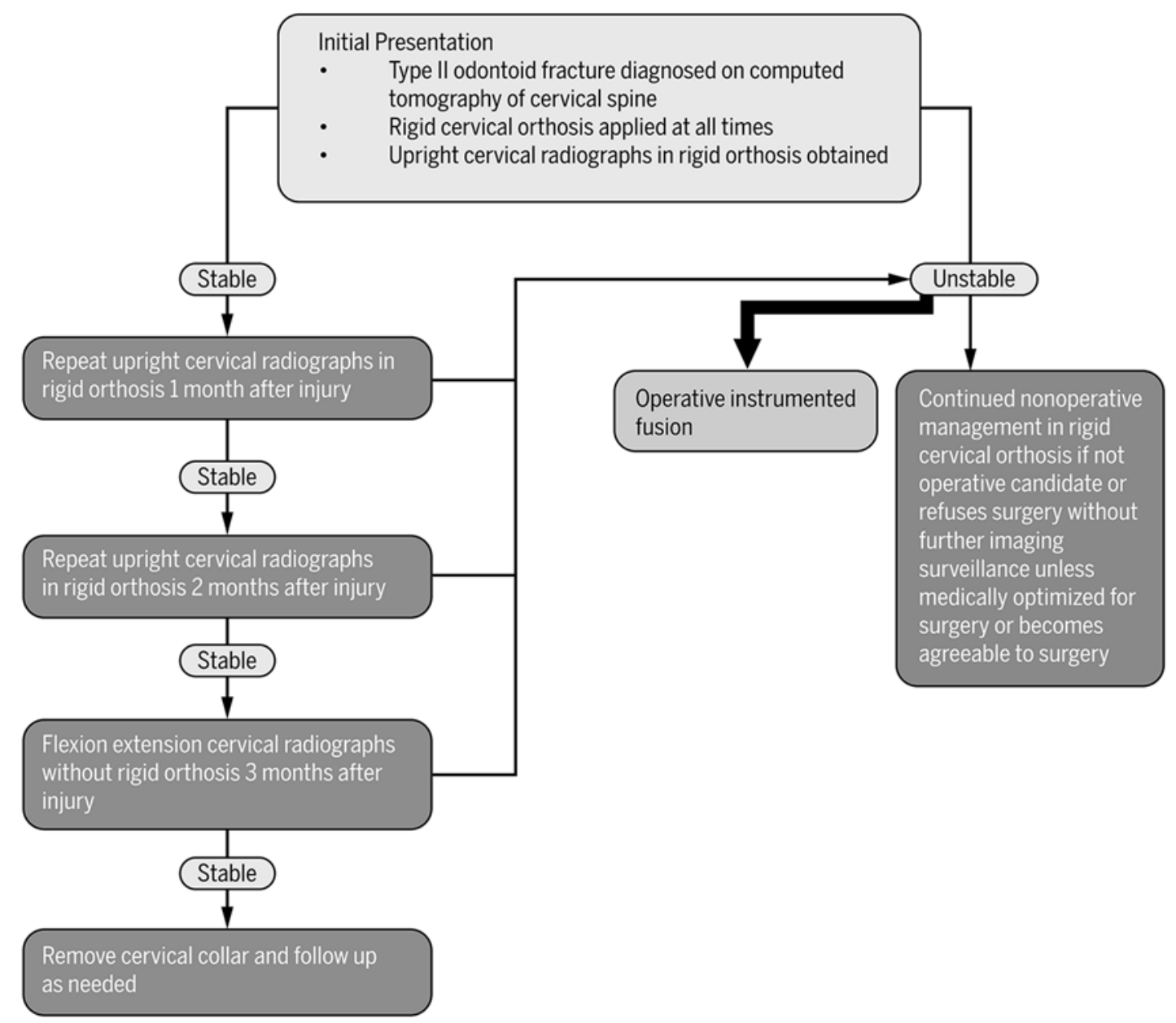

FIG. 2. Clinical flow diagram detailing patient follow-up and management. The bold arrow indicates preferred management when multiple management options exist. All patients were instructed to wear a rigid cervical orthosis for at least 3 months after injury.

tion who were managed nonoperatively due to severe medical or traumatic comorbidities, 7 (54\%) were found to have stability on follow-up radiographs, and instability remained in $6(46 \%)$, requiring delayed surgical fixation. Five of the 6 patients with persistent instability underwent a minimum of 3 months of observation with serial radiographs before operative intervention was performed. One patient was medically optimized for 3 weeks prior to operative intervention. There were no factors significantly associated with the development of stability over time after instability was demonstrated on initial upright imaging followed by a period of nonoperative management. Factors that were more common in those whose fractures remained unstable than in patients whose fractures became stable after initially unstable imaging included congestive heart failure, cerebrovascular disease, tobacco use, myelopathy, and fracture comminution, but these differences were nonsignificant. Displacement and fracture angulation were similar among these groups. Considering all fractures managed nonoperatively, $22 \%$ were unstable on follow-up imaging. We evaluated the association of stability on initial upright cervical radiographs in relation to follow-up stability (Table 2). This test demonstrated $89 \%$ sensitivity, indicating that $89 \%$ of patients who had stability on follow-up imaging exhibited stability on their initial upright radiographs. The PPV was $83 \%$, indicating that $83 \%$ of patients with stability on initial upright radiographs demonstrated stability thereafter. Specificity was only $35 \%$, which indicates that, of patients with fracture stability on follow-up, only $35 \%$ had instability on initial upright radiographs. Finally, the NPV was $46 \%$, indicating that $46 \%$ of patients with initial instability were unstable on follow-up imaging. This indicates that those with initial stability on upright imaging are likely to maintain the stability, and $54 \%$ of those with initial instability develop stability with nonoperative management.

Among patients managed nonoperatively, we identified factors associated with instability on follow-up (Table 3). Cervical myelopathy and cerebrovascular disease were associated with follow-up instability. All patients with cervical myelopathy managed nonoperatively were deemed to have instability, whereas only $19 \%$ of those without myelopathy demonstrated instability on follow-up. Consequently, cervical myelopathy on initial evaluation despite initial stable upright radiographs negatively correlated with follow-up stability (OR $0.04,95 \%$ CI $0.00-0.92$ ). 
TABLE 1. Baseline demographics of operative and nonoperative cohorts

\begin{tabular}{|c|c|c|}
\hline $\begin{array}{c}\text { Baseline } \\
\text { Characteristic }\end{array}$ & $\begin{array}{l}\text { Upfront Surgery } \\
\text { Cohort }(n=9)\end{array}$ & $\begin{array}{l}\text { Nonoperative } \\
\text { Cohort }(n=79)\end{array}$ \\
\hline Mean age, yrs* & 65 & 82 \\
\hline Upfront instability* & $9(100)$ & $13(16)$ \\
\hline Myelopathy* & $2(22)$ & $2(2.5)$ \\
\hline Median CCl & 4 & 5 \\
\hline Mean fracture displacement, $\mathrm{mm}^{*}$ & 6.75 & 1.75 \\
\hline Mean fracture angulation, ${ }^{\circ}$ & 12.3 & 8.1 \\
\hline Posterior/anterior/no displacement & $6 / 4 / 1$ & $31 / 9 / 39$ \\
\hline High-impact trauma & $2(22)$ & $13(16)$ \\
\hline Tobacco use & $6(67)$ & $28(35)$ \\
\hline Comminution & $1(11)$ & $6(7.6)$ \\
\hline Diabetes & $5(56)$ & $17(22)$ \\
\hline Cerebrovascular disease & $1(11)$ & $13(16)$ \\
\hline Moderate to severe renal disease & $2(22)$ & (11) \\
\hline
\end{tabular}

$\mathrm{CCl}=$ Charlson Comorbidity Index.

Values represent the number of patients (\%) unless stated otherwise.

* Variables for which there was a statistically significant difference between the upfront surgical and nonsurgical cohorts.

Cerebrovascular disease was significantly associated with lower odds of follow-up stability (OR 0.23 , 95\% CI $0.06-$ 1.0). Notably, increasing age demonstrated no association with follow-up instability (Fig. 3); $19.2 \%$ of patients older than 70 years and $20 \%$ of younger patients exhibited fracture instability on follow-up (OR 0.95, 95\% CI 0.18-5.0). A low-impact mechanism of injury demonstrated a trend toward more follow-up instability. No patient with a highimpact trauma severity managed nonoperatively developed delayed instability. Contrarily, $14 \%$ of patients who sustained low-velocity trauma such as ground-level falls managed in a cervical collar exhibited instability on serial imaging (OR 0.21, 95\% CI 0.01-3.7). Factors known to affect bone health, including tobacco use, diabetes, and renal disease, were not significantly associated with follow-up instability. All other historical factors were not significantly associated with instability.

Multiple imaging fracture characteristics were evaluated in relation to continued stability. Only dens displacement of $2 \mathrm{~mm}$ or more was significantly associated with follow-up instability (OR $0.29,95 \%$ CI $0.07-1.0)$. Twelve (32\%) of 37 patients with this degree of displacement had instability on follow-up. Only 5 (12\%) of the 41 patients without displacement developed instability. Notably, there was not a linear relationship between the magnitude of displacement and the prevalence of follow-up instability based on linear regression $\left(r^{2}=0.38\right)$. Posterior displacement $(n=31)$ of the odontoid peg was more common than anterior displacement $(n=9)$. However, the direction of displacement conferred no difference in follow-up stability (OR 1.22, 95\% CI 0.16-7.37). Twenty-nine percent and $33 \%$ of patients had follow-up instability with posterior and anterior displacement, respectively. When considering patients with posterior displacement alone, the mean length of displacement was similar between patients ex-
TABLE 2. Test characteristics of upright cervical radiographs with orthosis upon presentation

\begin{tabular}{cc}
\hline & Percentage \\
\hline Sensitivity & 89 \\
\hline Specificity & 35 \\
\hline PPV & 83 \\
\hline NPV & 46 \\
\hline
\end{tabular}

Described in relation to follow-up stability.

hibiting fracture stability and instability on follow-up (4 $\mathrm{mm}$ and $3.2 \mathrm{~mm}$, respectively). Sixteen patients demonstrated angulation of their fracture of $8^{\circ}$ or more on initial imaging. However, this was not associated with an increased odds of instability when compared with patients with less angulation (OR 1.07, 95\% CI 0.30-3.95). Posterior angulation was more common than anterior angulation (15 patients vs 1 patient), and 33\% of those with posterior angulation were deemed to have instability on follow-up, whereas the patient with anterior angulation maintained the stability. Posterior angulation was not associated with more instability than anterior angulation (OR 1.0, 95\% CI $0.0-42)$. Dens comminution was rare, only occurring in 6 patients, of whom only 1 developed delayed instability $(17 \%)$, which was similar to the prevalence of follow-up instability in those without comminution (19\%; OR 1.0, 95\% CI 0.0-61).

We compared the high-risk adverse event incidence found in the inpatient setting between those managed with early surgical fixation and cervical bracing (Table 4). When considering adverse events aggregately, $33 \%$ of patients managed surgically during their presenting encounter and $8.9 \%$ of those managed nonoperatively had one or more complication $(\mathrm{p}=0.03)$. Respiratory failure was the most common complication $(n=5)$, followed by pneumonia $(n=3)$, urinary tract infection $(n=2)$, acute kidney injury $(\mathrm{n}=2)$, deep venous thrombosis $(\mathrm{n}=2)$, and pulmonary embolism $(n=1)$. Respiratory failure requiring ventilation was more common among patients managed

TABLE 3. Associations with follow-up instability and stability

\begin{tabular}{|c|c|}
\hline & OR $(95 \% \mathrm{Cl})$ \\
\hline \multicolumn{2}{|l|}{ Associations w/ follow-up instability } \\
\hline Cervical myelopathy* & $0.04(0.0-0.92)$ \\
\hline Cerebrovascular disease $^{*}$ & $0.23(0.06-1.0)$ \\
\hline Dens displacement $\geq 2 \mathrm{~mm}^{*}$ & $0.29(0.07-1.0)$ \\
\hline \multicolumn{2}{|l|}{ Associations w/ follow-up stability } \\
\hline Stability on initial upright radiographs* & $4.29(1.0-18)$ \\
\hline \multicolumn{2}{|c|}{ Notable factors not associated w/ stability or instability } \\
\hline Age $>70$ yrs & $0.95(0.18-5.0)$ \\
\hline Low-impact injury & $0.21(0.01-3.7)$ \\
\hline Tobacco use & $1.01(0.29-3.8)$ \\
\hline Diabetes & $0.86(0.21-4.3)$ \\
\hline Renal disease & $0.29(0.05-1.7)$ \\
\hline
\end{tabular}

* Variables for which there was a statistically significant association. 


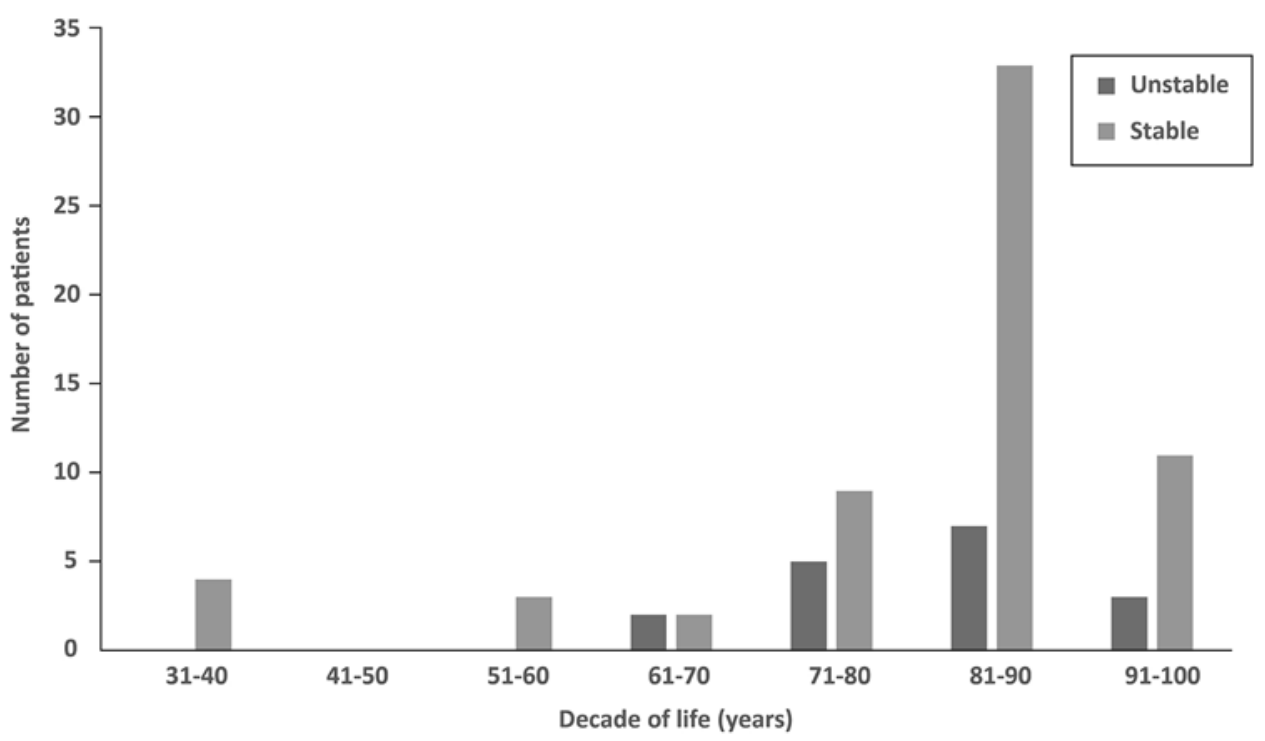

FIG. 3. Bar graph showing the association of age with follow-up instability in type II odontoid fractures.

with upfront surgery (33\% vs $3 \%, \mathrm{p}=0.007)$. Examined individually, no other complication was significantly more common in either cohort. Table 5 summarizes delayed complications among patients who underwent upfront and delayed operative management as well as nonoperative management during their associated follow-up periods. No patient had late neurological deterioration that was attributable to their odontoid fracture regardless of management chosen throughout follow-up, including 6 patients with more than 12 months of follow-up. The only late complication identified was hardware failure in a male smoker 3.5 years after delayed surgical fixation, complicated by surgical site infection. He was offered revision of his construct but refused and had no complaints 6 months later.

Neither operative nor nonoperative management conferred a significant difference in length of hospital or ICU stay, discharge disposition, or 30-day mortality. The mean ICU LOSs were 4.8 days and 5.7 days in the operative and nonoperative cohorts, respectively. The mean hospital LOSs were 5.8 days and 5.2 days in operative and non-

TABLE 4. Comparison of outcomes in patients managed with upfront surgery and with external orthosis

\begin{tabular}{|c|c|c|}
\hline Outcome & $\begin{array}{c}\text { Upfront } \\
\text { Surgery }(n=9)\end{array}$ & $\begin{array}{l}\text { Nonoperative } \\
\text { Cohort }(n=79)\end{array}$ \\
\hline Follow-up instability* & $0(0)$ & $17(22)$ \\
\hline In-hospital adverse event* & $3(33)$ & $7(8.9)$ \\
\hline Respiratory failure* & $3(33)$ & $2(3)$ \\
\hline 30-day mortality & $0(0)$ & $4(5)$ \\
\hline Mean hospital LOS, days & 5.8 & 5.2 \\
\hline Mean ICU LOS, days & 4.8 & 5.7 \\
\hline Rehabilitation disposition & $4(44)$ & $45(57)$ \\
\hline
\end{tabular}

operative groups, respectively. No significant difference in disposition was noted between cohorts; $56 \%$ of those managed operatively and $42 \%$ of nonoperative patients were discharged home. The remaining patients required rehabilitation. Thirty-day mortality was rare, occurring in only $5 \%$ of nonoperatively managed patients and none of those managed operatively. All patients who died within 30 days had high injury severity scores and poor prognostic indicators from systemic injuries.

\section{Discussion}

Although type II odontoid fracture management has been controversial, we found that patients can often be managed safely with an external orthosis, as corroborated by several recent reports, in contrast to previously raised concerns. ${ }^{8,10,12,16,17,20}$ Cervical myelopathy, cerebrovascular disease, and fracture displacement were associated with follow-up instability. Patients with these factors may benefit from early surgery. However, even after a trial of nonoperative therapy, we found no added morbidity when delayed instability occurred. Most fractures either remained stable or became stable on follow-up imaging, negating the need for surgical fixation. Stability observed on the initial upright cervical radiographs portended a high likelihood of continued stability. Even with initial radiographs showing instability, more than half of the patients exhibited stability on follow-up presumably via fibrous union. Finally, the incidence of perioperative and delayed adverse events was higher among patients managed surgically despite a generally younger and healthier surgical cohort.

Our study population was similar to those in previous reports with some exceptions. ${ }^{1}$ Our patients were generally older and sustained less high-velocity trauma than those in previous reports. , $^{2,14}$ The association of geriatric type II odontoid fractures with low-velocity trauma may result in delayed immobilization and higher nonunion rates. ${ }^{14}$ Neurological deficits, including myelopathy on presentation, occurred in only $5 \%$ of our patients, whereas others 
TABLE 5. Delayed complications during follow-up among patients managed operatively and nonoperatively

\begin{tabular}{lcccc}
\hline \multicolumn{1}{c}{ Outcome } & $\begin{array}{c}\text { Upfront } \\
\text { Surgery }(\mathrm{n}=9)\end{array}$ & $\begin{array}{c}\text { Delayed } \\
\text { Surgery }(\mathrm{n}=8)\end{array}$ & $\begin{array}{c}\text { Unstable Nonop } \\
\text { Management }(\mathrm{n}=9)\end{array}$ & $\begin{array}{c}\text { Stable Nonop } \\
\text { Management }(\mathrm{n}=62)\end{array}$ \\
\hline Mean follow-up, mos & 4.5 & 14.6 & 4.5 & 4.6 \\
\hline Delayed neurological deterioration, $\mathrm{n}$ & 0 & 0 & 0 & 0 \\
\hline Delayed nonneurological complications, $\mathrm{n}$ & 0 & $2^{*}$ & 0 & 0 \\
\hline
\end{tabular}

Delayed complication was defined as occurring more than 30 days after injury if managed nonoperatively or more than 30 days after surgery if managed operatively.

* Surgical site infection requiring wound revision and subsequent hardware failure 3.5 years after injury in the same patient.

have reported that $7.5 \%-33 \%$ of patients presented with deficits. ${ }^{1,3,9,11,20}$ More recent articles have indicated rates of neurological deficits similar to ours, which may reflect improvements in early immobilization. ${ }^{2}$ Ten percent in our population underwent primary surgical fixation, whereas $90 \%$ were managed nonoperatively, which is similar to other series. ${ }^{1,9,14}$ All patients managed surgically achieved follow-up stability, which is similar to that of previous reports. ${ }^{1,9,10,14}$ Our rate of delayed instability is similar to previous literature, which indicated instability of $22 \%-$ $36 \%$. ${ }^{1,8,14,19}$ Higher union rates in ours and other contemporary reports may reflect improved medical management of osteoporosis and improved orthotic technology over time. ${ }^{11,20}$

Cervical upright radiographs while wearing a brace are critical for the evaluation of type II odontoid fractures at our institution. To our knowledge, the value of this test in relation to long-term stability has not been studied. We identified this test to have excellent PPV and sensitivity for detecting long-term stability. Previously, upright radiographs as part of a protocol including CT and MRI have been evaluated in which no abnormality identified using this protocol was associated, with a 91\% NPV of acute instability. ${ }^{22}$ However, long-term stability was not assessed. Notably, CT outperforms plain radiography, and plain radiography should not be used alone during the initial diagnostic phase of care. ${ }^{23,24}$ Validated standardized indicators of instability have not been identified and are a worthwhile future endeavor. ${ }^{18,25}$

Cervical myelopathy and cerebrovascular disease were associated with delayed instability among nonoperatively managed patients. We and others agree that any neurological deficit noted at presentation related to a type II odontoid fracture warrants primary surgical fixation. ${ }^{1}$ Our finding of an association of myelopathy with nonunion contradicts class II evidence that found no such association. ${ }^{21}$ However, class III evidence has demonstrated an up to threefold higher prevalence of nonunion among patients with neurological deficits compared with neurologically intact patients with type II odontoid fractures. ${ }^{1,4,8}$ Cerebrovascular disease has not been previously evaluated as a contributor to nonunion but may reflect disruption of a vascular watershed at the base of the dens, resulting in poorer healing. ${ }^{10-12}$

Notably, in our adult study population we did not identify an association between older age and nonunion, unlike previous reports. ${ }^{1,3,4,7,9-11,14,21}$ Some reports only considered bony union an acceptable outcome, which is less common in geriatric type II odontoid fractures. ${ }^{12,14} \mathrm{We}$ propose that bony union is unnecessary so long as fibrous union is achieved. Fibrous union may not correlate with age. Addi- tionally, articles that included children, who have excellent fusion despite nonoperative management, have generally corroborated the traditional association of increasing age with nonunion. ${ }^{1}$ Furthermore, contemporary reports are similar in demonstrating no association between age and nonunion, whereas earlier reports are contradictory. ${ }^{2,4,9,14}$ Improved medical osteoporosis and comorbidity management likely mitigate the association of age and nonunion.

Fracture displacement was associated with an elevated incidence of follow-up instability in our cohort. Displacement has repeatedly been correlated with nonunion. ${ }^{2,4,7}$, 9,14 Direction of displacement has been an important consideration for some authors who have reported posterior displacement as a risk factor for chronic instability. ${ }^{2-4,7,14}$ However, we and others did not find direction to be significantly associated with nonunion. ${ }^{10}$ The magnitude of displacement did not confer higher nonunion risk on our analysis, contrary to earlier reports. ${ }^{4,7,9,14,26}$ Some authors have claimed that increasing magnitude of anterior displacement does not confer higher nonunion rates, whereas increasing posterior displacement does., ${ }^{71}$ Our experience does not support either postulation. The lower limit for significant displacement has not been standardized. We defined displacement of $2 \mathrm{~mm}$ or more as significant in effort to be as conservative as possible in selecting patients to be managed nonoperatively. Others have endorsed using 3-6 $\mathrm{mm}$ of displacement to define significant displacement. ${ }^{1,2}$, 9,10,14 Furthermore, we identified no association between comminution and nonunion. This may reflect type II error due to the rarity of comminution in our population. Others have endorsed up to $100 \%$ nonunion in comminuted fractures, recommending their early operative fixation..$^{1-3,27}$

Our findings support previous studies that showed that patients who were managed operatively during their initial encounter were at higher risk of complications. ${ }^{6,13,14,28}$ Previous literature indicated the opposite, associating higher morbidity of nonoperative therapy with prolonged bedrest. ${ }^{8}$ Early external orthoses included halo vest application, which conferred elevated morbidity and mortality so much that Majercik and colleagues coined this treatment a "death sentence." 29 Contemporary cervical rigid orthoses mitigate the morbidity associated with bedrest and have fewer and less severe complications. ${ }^{14,19,30}$ Conversely, surgical complications have remained constant and are generally more severe: ${ }^{13} 69 \%$ of perioperative complications have been classified as severe. ${ }^{13}$ Complication incidence and severity should be considered jointly. Respiratory complications following surgical interventions were most common in our series and in others. ${ }^{4,6,8,10}$ Consistent with two large series of nearly 4000 patients, we did not dem- 
onstrate significant differences in individual complication rates between surgical and nonsurgical cohorts, aside from respiratory failure. ${ }^{5,6}$ Despite our surgical cohort being generally younger and healthier, the ICU and hospital LOSs were similar regardless of management. ICU and hospital LOSs have been reported to be longer in surgically treated patients. ${ }^{4,8,10,13,14,21,28}$

Furthermore, due to a higher prevalence of comorbidities and advanced age, nonoperative patients are expected to have elevated risk of need for rehabilitation when compared with operatively managed patients. However, we found similar rates of rehabilitation dispositions among both cohorts. Finally, none of our patients managed nonoperatively had new neurological deficits, even when bony union was not achieved, highlighting the safety of fibrous union. Hanigan et al. and others corroborated this safety and indicated that $75 \%$ of those with fibrous union returned to their baseline neurological status, indicating that fibrous union does not negatively impact QOL. ${ }^{8,10,12,19}$

There are several limitations to our study. First, we did not include QOL data. This will be a focus of future prospective study with longer follow-up to evaluate the long-term QOL effect of fibrous union. Second, our study is hindered by selection bias of operative versus nonoperative management. ${ }^{30}$ With good reason, surgeons operate on healthier patients, which likely confers a higher likelihood of treatment success in operative patients. Despite this potential bias to favor surgical patient outcomes, we and others observed similar mortality and higher morbidity associated with surgery, which may be considered a reason to strongly consider nonoperative therapy when possible. ${ }^{5,6} \mathrm{~A}$ well-designed randomized controlled trial is necessary and has been protocolized, but results are yet to be published. ${ }^{31}$ Finally, long-term follow-up is limited in this report, which is a common critique in trauma literature and is expected, considering our 3-month management algorithm.

\section{Conclusions}

To our knowledge, we provide the first study to detail the validity of upright cervical radiographs at presentation in association with follow-up stability on serial radiographs of type II odontoid fractures. Second, we highlight factors associated with delayed instability, including cervical myelopathy, cerebrovascular disease, and fracture displacement. Our findings do not support the association of geriatric type II odontoid fractures with nonunion indicated previously. Complications were more common in our operative cohort than in our nonoperative cohort. Finally, fibrous union, which may occur with nonoperative management, provides adequate stability, at least in shortterm follow-up.

\section{Acknowledgments}

The Indiana University Department of Neurological Surgery provided $\$ 260$ for the statistical analysis required for this paper. The statistician was granted authorship for his significant time in analysis of the raw data. The funding source was not involved in study design, study conduction, data collection, data analysis, data interpretation, data management, manuscript preparation, manuscript review, or manuscript approval.

\section{References}

1. Anderson LD, D'Alonzo RT. Fractures of the odontoid process of the axis. J Bone Joint Surg Am. 1974;56(8):1663-1674.

2. Hadley MN, Dickman CA, Browner CM, Sonntag VK. Acute axis fractures: a review of 229 cases. J Neurosurg. 1989;71(5 Pt 1):642-647.

3. Iyer S, Hurlbert RJ, Albert TJ. Management of odontoid fractures in the elderly: a review of the literature and an evidencebased treatment algorithm. Neurosurgery. 2018;82(4):419-430.

4. Ekong CE, Schwartz ML, Tator CH, et al. Odontoid fracture: management with early mobilization using the halo device. Neurosurgery. 1981;9(6):631-637.

5. Vaccaro AR, Kepler CK, Kopjar B, et al. Functional and quality-of-life outcomes in geriatric patients with type-II dens fracture. J Bone Joint Surg Am. 2013;95(8):729-735.

6. Dhall SS, Yue JK, Winkler EA, et al. Morbidity and mortality associated with surgery of traumatic $\mathrm{C} 2$ fractures in octogenarians. Neurosurgery. 2017;80(6):854-862.

7. Schatzker J, Rorabeck CH, Waddell JP. Fractures of the dens (odontoid process). An analysis of thirty-seven cases. J Bone Joint Surg Br. 1971;53(3):392-405.

8. Hanigan WC, Powell FC, Elwood PW, Henderson JP. Odontoid fractures in elderly patients. J Neurosurg. 1993;78(1):32-35.

9. Apuzzo ML, Heiden JS, Weiss MH, et al. Acute fractures of the odontoid process. An analysis of 45 cases. J Neurosurg. 1978;48(1):85-91.

10. Hadley MN, Browner C, Sonntag VK. Axis fractures: a comprehensive review of management and treatment in 107 cases. Neurosurgery. 1985;17(2):281-290.

11. Polin RS, Szabo T, Bogaev CA, et al. Nonoperative management of types II and III odontoid fractures: the Philadelphia collar versus the halo vest. Neurosurgery. 1996;38(3):450-457.

12. Ryan MD, Taylor TK. Odontoid fractures in the elderly. $J$ Spinal Disord. 1993;6(5):397-401.

13. Patterson JT, Theologis AA, Sing D, Tay B. Anterior versus posterior approaches for odontoid fracture stabilization in patients older than 65 years: 30 -day morbidity and mortality in a national database. Clin Spine Surg. 2017;30(8):E1033-E1038.

14. Dunn ME, Seljeskog EL. Experience in the management of odontoid process injuries: an analysis of 128 cases. Neurosurgery. 1986;18(3):306-310.

15. Molinari RW, Dahl J, Gruhn WL, Molinari WJ. Functional outcomes, morbidity, mortality, and fracture healing in 26 consecutive geriatric odontoid fracture patients treated with posterior fusion. J Spinal Disord Tech. 2013;26(3):119-126.

16. Hart R, Saterbak A, Rapp T, Clark C. Nonoperative management of dens fracture nonunion in elderly patients without myelopathy. Spine (Phila Pa 1976). 2000;25(11):1339-1343.

17. Molinari RW, Khera OA, Gruhn WL, McAssey RW. Rigid cervical collar treatment for geriatric type II odontoid fractures. Eur Spine J. 2012;21(5):855-862.

18. Bono CM, Vaccaro AR, Fehlings M, et al. Measurement techniques for upper cervical spine injuries: consensus statement of the Spine Trauma Study Group. Spine (Phila Pa 1976). 2007:32(5):593-600.

19. Charlson ME, Pompei P, Ales KL, MacKenzie CR. A new method of classifying prognostic comorbidity in longitudinal studies: development and validation. J Chronic Dis. 1987; 40(5):373-383.

20. Waqar M, Van-Popta D, Barone DG, Sarsam Z. External immobilization of odontoid fractures: a systematic review to compare the halo and hard collar. World Neurosurg. 2017;97: 513-517.

21. Lennarson PJ, Mostafavi H, Traynelis VC, Walters BC. Management of type II dens fractures: a case-control study. Spine (Phila Pa 1976). 2000;25(10):1234-1237.

22. Patel MB, Humble SS, Cullinane DC, et al. Cervical spine collar clearance in the obtunded adult blunt trauma patient: a systematic review and practice management guideline from 
the Eastern Association for the Surgery of Trauma. J Trauma Acute Care Surg. 2015;78(2):430-441.

23. Holmes JF, Akkinepalli R. Computed tomography versus plain radiography to screen for cervical spine injury: a metaanalysis. J Trauma. 2005;58(5):902-905.

24. Osterhoff G, Schnake K, Scheyerer MJ, et al. Recommendations for diagnosis and treatment of odontoid fractures in geriatric patients. Z Orthop Unfall. 2020;158(6):647-656.

25. Bono CM, Schoenfeld AJ, Anderson PA, et al. Observer variability of radiographic measurements of $\mathrm{C} 2$ (axis) fractures. Spine (Phila Pa 1976). 2010;35(12):1206-1210.

26. Clark CR, White AA III. Fractures of the dens. A multicenter study. J Bone Joint Surg Am. 1985;67(9):1340-1348.

27. Grauer JN, Shafi B, Hilibrand AS, et al. Proposal of a modified, treatment-oriented classification of odontoid fractures. Spine J. 2005;5(2):123-129.

28. Chapman J, Smith JS, Kopjar B, et al. The AOSpine North America Geriatric Odontoid Fracture Mortality Study: a retrospective review of mortality outcomes for operative versus nonoperative treatment of 322 patients with long-term followup. Spine (Phila Pa 1976). 2013;38(13):1098-1104.

29. Majercik S, Tashjian RZ, Biffl WL, et al. Halo vest immobilization in the elderly: a death sentence? J Trauma. 2005;59(2): $350-358$.

30. Schroeder GD, Kepler CK, Kurd MF, et al. A systematic review of the treatment of geriatric type II odontoid fractures. Neurosurgery. 2015;77(suppl 1):S6-S14.

31. Huybregts JGJ, Jacobs WCH, Peul WC, Vleggeert-Lankamp CLA. Rationale and design of the INNOVATE Trial: an international cooperative study on surgical versus conservative treatment for odontoid fractures in the elderly. BMC Musculoskelet Disord. 2014;15:7.

\section{Disclosures}

Mr. Eckert acknowledges payment from the Indiana University Department of Neurological Surgery for statistical analysis.

\section{Author Contributions}

Conception and design: Wilson, Hoyos, Huh, Priddy, Avila, Mendenhall, Anokwute, Stockwell. Acquisition of data: all authors. Analysis and interpretation of data: Wilson, Hoyos, Huh, Priddy, Mendenhall, Anokwute, Eckert, Stockwell. Drafting the article: Wilson, Hoyos, Huh, Priddy, Mendenhall, Anokwute, Stockwell. Critically revising the article: Wilson, Stockwell. Reviewed submitted version of manuscript: all authors. Approved the final version of the manuscript on behalf of all authors: Wilson. Statistical analysis: Wilson, Hoyos, Huh, Priddy, Mendenhall, Anokwute, Eckert, Stockwell. Administrative/ technical/material support: all authors. Study supervision: all authors.

\section{Supplemental Information}

\section{Previous Presentations}

This study was presented orally at the 36th Annual Meeting of the AANS/CNS Section on Disorders of the Spine and Peripheral Nerves Spine Summit 2020, Las Vegas, Nevada, March 8, 2020.

\section{Correspondence}

Christopher Wilson: Indiana University School of Medicine, Indianapolis, IN. wilsochd@iu.edu. 